\title{
Pesquisa de depresión mayor en hipertensos consultantes de la Atención Primaria
}

\author{
Angelina Dois C ${ }^{1 a}$, Angélica Cazenave $2 a$. \\ Frequency of depression among \\ hypertensive subjects \\ in a primary care clinic
}

\begin{abstract}
Background: Severe depression and hypertension are two common conditions in the Chilean population. There is a possibility that the coexistence of these diseases is not a coincidence and that hypertension can be pathogenically related to depression. Aim: To assess the frequency of depression among hypertensive subjects consulting in a primary care clinic. Patients and methods: Beck depression inventory, a sociodemographic survey and a risk scale for depression elaborated by the Chilean Ministry of Health were applied to 442 hypertensive patients consulting in a public primary care clinic. Results: According to Beck depression inventory score, $53 \%, 33 \%$ and $14 \%$ of subjects had a mild, moderate or severe depression, respectively. Most individuals were housewives with incomplete primary education. There was a significant association between depression scores, family Apgar score, mood perception, family violence, alcohol consumption and smoking. Conclusions: There is a high frequency of depression among adults with hypertension consulting in a public primary care clinic (Rev Méd Chile 2009; 137: 475-480).
\end{abstract}

(Key words: Depression; Hypertension; Psychological tests)

Recibido el 18 de junio, 2008. Aceptado el 23 de diciembre, 2008.

${ }^{1}$ Departamento de Salud del Adulto y Senescente, Escuela de Enfermería, Pontificia Universidad Católica de Chile. ${ }^{2}$ Departamento de Salud del Niño y Adolescente, Escuela de Enfermería, Pontificia Universidad Católica de Chile. Santiago de Chile.

aEnfermera-Matrona

T a Encuesta Nacional de Salud 2003 muestra un ـcambio en el perfil epidemiológico del país con un aumento de las patologías crónicas no transmisibles del adulto entre las que se encuentra

Correspondencia a: E.M. Angelina Dois C. Departamento de Salud del Adulto y Senescente, Escuela de Enfermería, Pontificia Universidad Católica de Chile. Av. Vicuña Mackena 4860. Macul, Santiago, Chile. Fono: 56-2-3545837. Fax: 56-2-3547025. E mail: adois@uc.cl la hipertensión arterial y la depresión mayor ${ }^{1}$. La hipertensión arterial (HTA) ha sido considerada por el Ministerio de Salud como una condición de salud prioritaria desde 1997, tanto por su magnitud como por su asociación con los índices de morbimortalidad cardiovascular, es además la enfermedad crónica más común en Chile, su prevalencia en población general es de 33,7\%, siendo ésta mayor en hombres que en mujeres $(36,7 \%$ $30,8 \%$, respectivamente) ${ }^{1}$, con una tasa de mortalidad ajustada por edad y sexo de 15/100.000 y proyectada para 2010 en 16,5/100.000². Según la

Artículo de 
misma encuesta, $60 \%$ de las personas a las que se les detectó HTA conocían su condición, pero sólo $36 \%$ estaba en tratamiento y de ellos 33\% estaba normotenso ${ }^{1}$. El estudio de carga de enfermedad realizado en Chile mostró que la enfermedad hipertensiva produjo una pérdida de años de vida saludables equivalentes a 4,4 AVISA por mil habitantes, lo que se traduce también en una carga importante para el nivel primario de salud, siendo la hipertensión arterial la primera causa de consulta en el nivel primario de atención $(9,4 \%$ de todas las consultas) ${ }^{3}$.

Por otro lado, la depresión mayor (DM) muestra una prevalencia en población general mayor de 15 años entre 7,5\% y 10\% siendo el grupo más afectado el que se encuentra entre 20 y 45 años, con una relación 2:1 en población femenina sobre la masculina ${ }^{4,5}$. Del total de los consultantes en atención primaria, 30\% tiene cuadros depresivos y estas tasas aumentan en personas que tienen además enfermedades físicas de gravedad ${ }^{4}$. El $75 \%$ de las personas con depresión consultan por primera vez en la atención primaria con una tasa de reconocimiento inferior a $60 \%$ y menos de $30 \%$ de las personas recibe tratamiento; y de las que lo reciben, menos de $20 \%$ lo prolonga por el tiempo recomendado 4 . Situación que se agrava al observar que la depresión mayor es la segunda causa de pérdida de años de vida saludable y la primera causa de discapacidad en las mujeres ${ }^{6}$.

En Chile, tanto las tasas de prevalencia de depresión como de enfermedad coronaria constituyen dos de los más relevantes problemas de salud pública. La depresión mayor es una patología fuertemente ligada a enfermedades cardiovasculares $^{7}$, siendo la comorbilidad entre ambas fuente de una gran cantidad de enfermedades crónicas y degenerativas con altas tasas de mortalidad a nivel $\mathrm{global}^{8}$. De esta forma, una persona que evoluciona con un síndrome depresivo después de los 40 años de edad debe ser evaluada para estimar su riesgo de enfermedad coronaria junto a la planificación e implementación de un plan de tratamiento precoz de su depresión, así como la pesquisa de otros factores de riesgo cardiovascular ${ }^{8}$.

Respecto a la asociación entre ambos cuadros, se han postulado diferentes mecanismos, entre ellos, los relativos a la hiperactividad simpática adrenérgica y a procesos inflamatorios crónicos producidos por la DM que aumentan la reactividad plaquetaria $9,10,12,13$ y los que relacionan la depresión con estilos de vida poco saludables asociado con una dieta pobre, tabaquismo, aumento del consumo de alcohol, sedentarismo y baja adherencia al tratamiento antihipertensivo, todos factores que aumentan el riesgo de enfermedad cardiovascular ${ }^{10,11,13}$.

Debido al aumento epidemiológico y a la gravedad de ambas entidades clínicas, el objetivo general de este estudio fue determinar la incidencia de depresión en consultantes de la atención primaria con hipertensión arterial crónica, su perfil sociodemográfico y de factores de riesgo asociados para depresión mayor e hipertensión arterial.

\section{MATERIAL Y MÉTODO}

Tipo y diseño del estudio. Estudio analítico de corte transversal que evaluó sintomatología depresiva y perfil sociodemográfico de los participantes.

Selección y tamaño de la muestra. El universo comprendió a todos los adultos mayores de 18 años del Programa Cardiovascular de un Centro de Salud Familiar (CESFAM) de Puente Alto, equivalentes a 903 personas adultas de ambos sexos, en control entre septiembre de 2006 y marzo de 2007. La muestra quedó constituida por 442 usuarios de ambos sexos mayores de 18 años, que aceptaron participar voluntariamente del estudio y a quienes se les solicitó firmar consentimiento informado. Los criterios de exclusión fueron: embarazadas o puérperas, patología psiquiátrica 0 neurológica descompensada o invalidante, hospitalizaciones o duelos de menos de 6 meses al aplicar el instrumento o con depresión mayor diagnosticada o en tratamiento.

Aplicación del instrumento. El instrumento fue aplicado por encuestadores capacitados para esos fines, previa firma de un Consentimiento Informado. El instrumento de evaluación estuvo formado a su vez por tres instrumentos, el primero sobre antecedentes sociodemográficos, el segundo por la Escala de Factores de Riesgo propuesta por el Ministerio de Salud (MINSAL) en la Guía Clínica La Depresión $^{4}$ y el tercero por el Inventario de 
Depresión de Beck [IDB] adaptado al castellano y validada por Vázquez y Sanz ${ }^{14}$ que permite cuantificar la sintomatología y evaluar la gravedad (intensidad sintomática) de la depresión y que ha sido utilizada en otros grupos poblacionales con propósitos de screening ${ }^{15-17}$. Este último, solo aplicado a quienes tuvieran al menos 1 punto en la Escala de Factores de Riesgo propuesta por el MINSAL.

Los resultados se analizaron a partir de medidas de tendencia central y análisis correlacional de las variables usando el programa estadístico SPSS 12.0.

Consideraciones éticas. Las consideraciones éticas de este estudio se originan a partir del resguardo de la dignidad de la persona durante toda investigación, considerando el consentimiento informado aprobado para el Proyecto de Investigación № 02/ 2006 que asegura la confidencialidad de la información, la participación voluntaria y el respeto por la autonomía de las enfermeras participantes. El Proyecto de Investigación y el Consentimiento Informado fueron aprobados por el Comité de Ética de la Escuela de Enfermería de la Pontificia Universidad Católica de Chile.

\section{RESULTADOS}

En el estudio realizado, se encontró que de la muestra conformada por 442 usuarios, 196 pacientes obtuvieron menos de 1 punto en la Escala de Factores de Riesgo del MINSAL, equivalente a $44,3 \%$ del total, lo que sugiere que no cursaban en ese momento con un cuadro depresivo. De los 246 pacientes restantes, equivalentes a $55,7 \%$ del total, 42 usuarios correspondiente a $17,1 \%$ del total obtuvieron más de 1 punto en la misma
Escala, sin embargo obtuvieron puntajes menores a 9 puntos medidos según Inventario de Depresión de Beck, lo que sugiere ausencia de depresión clínica.

Por otra parte, 204 usuarios es decir $46,2 \%$ de los participantes en la investigación, obtuvieron más de 1 punto en la Escala del MINSAL y más de 9 puntos en el Inventario de Depresión de Beck, lo que sugiere un cuadro clínico de depresión. En este grupo, $76,5 \%$ fueron mujeres y $23,5 \%$ hombres, de ellos $68,2 \%$ se encontraban en un rango de edad entre 55 y 74 años, $59,1 \%$ era casado y $20,2 \%$ viudo. Según el nivel de educación, la muestra se distribuyó normalmente con mayor proporción de personas con educación básica incompleta $(31,6 \%)$ y media completa $(17,4 \%)$. Según el tipo de ocupación, 55,56\% realizaba labores de dueña de casa, 28,2\% era trabajador activo y 13,9\% jubilado. Según previsión, 31,6\% registraba FONASA B.

La distribución del total de usuarios que tuvieron puntajes sugerentes de depresión según el Inventario de Depresión de Beck mostró que 52,45\% presentaba depresión leve, 33,33\% depresión moderada y $14,21 \%$ depresión severa. Todos los usuarios fueron derivados a sus médicos tratantes para el inicio de un plan terapéutico (Tabla 1).

El análisis no paramétrico de las variables se realizó con las pruebas de Kendall y Spearman encontrándose una correlación significativa entre el índice de depresión de Beck y el puntaje del APGAR familiar ( $p<0,05)$, percepción del estado del ánimo $(p<0,01)$, presencia de violencia intrafamiliar ( $p<0,01)$ y consumo de alcohol $(p<0,01$ ).

A su vez el puntaje del APGAR familiar presentó correlación estadísticamente significativa

Tabla 1. D istribución de casos según ID B y sexo

\begin{tabular}{|c|c|c|c|c|}
\hline \multirow[t]{2}{*}{ Puntaje ID B } & \multicolumn{2}{|c|}{ Sexo } & \multirow[t]{2}{*}{$\mathrm{N} \cong$ de casos } & \multirow[t]{2}{*}{ Porcentaje } \\
\hline & Mujer & Hombre & & \\
\hline $0-9$ & 29 & 13 & 42 & 17,1 \\
\hline $10-18$ & 77 & 30 & 107 & 43,5 \\
\hline $19-29$ & 61 & 7 & 68 & 27,6 \\
\hline \multirow{2}{*}{$>30$} & 21 & 8 & 29 & 11,8 \\
\hline & 188 & 58 & 246 & 100 \\
\hline
\end{tabular}

Artículo de 
$(p<0,05)$ con percepción del estado de ánimo y con la presencia de violencia intrafamiliar, la que a su vez también se correlacionó significativamente con la ocurrencia de violencia laboral ( $p<0,01$ ), consumo de tabaco $(\mathrm{p}<0,01)$ y alcohol $(\mathrm{p}<0,05)$ y con el uso de tranquilizantes $(p<0,05)$ (Figura 1 ).

\section{Discusión}

En Chile, por su prevalencia, tanto la depresión mayor como la hipertensión arterial se constituyen como dos problemas de salud pública relevantes. La incidencia de depresión mayor que presentó la población hipertensa del presente estudio fue de $51 \%$, lo que concuerda con las cifras encontradas por otros investigadores ${ }^{18}$. Además, los datos muestran que en este grupo la incidencia de depresión fue mayor en mujeres que en hombres, lo que se ajusta a la realidad nacional ${ }^{4}$, sin embargo, esta relación se modificó en todas las categorías ajustándose de manera global de 2:1 a 3:1 mujeres/hombres.

Si se consideran estos dos indicadores y se analizan a la luz de la evidencia científica disponible que demuestra que la asociación de ambas enfermedades está ligada a un mayor desarrollo de enfermedades cardiovasculares ${ }^{7,11,19,20}$, claramente se puede afirmar que este grupo de pacientes se constituyen como grupo de riesgo relevante y que debe ser considerado al momento de planificar intervenciones de promoción y prevención, presentando especial importancia la pesquisa y diagnóstico precoz.

Es por lo anterior que se hace relevante el considerar la evaluación del estado del ánimo de aquellas personas adultas que cursan hipertensión arterial, en especial a aquellos con dificultades en el control de la misma y de adherencia al tratamiento médico ${ }^{8}$, para estimar con mayor certeza el riesgo de enfermedad coronaria junto con la pesquisa de otros factores de riesgo cardiovascular, lo que permitiría la instauración de un plan de tratamiento precoz para el cuadro depresivo.

Una herramienta eficiente para la detección de depresión mayor en estos grupos es incluir en la atención habitual de los pacientes del programa cardiovascular, la Escala de Factores de Riesgo para la pesquisa de depresión propuesta por el Ministerio de Salud en su Guía Clínica ${ }^{4}$ y que considera responder a tres preguntas de carácter dicotómico con la que se detecta con facilidad y rapidez la existencia de síntomas como la disminución del ánimo y anhedonia en los consultantes, ambas condiciones necesarias para el diagnóstico de depresión. La simplicidad de este instrumento permite que cualquier profesional

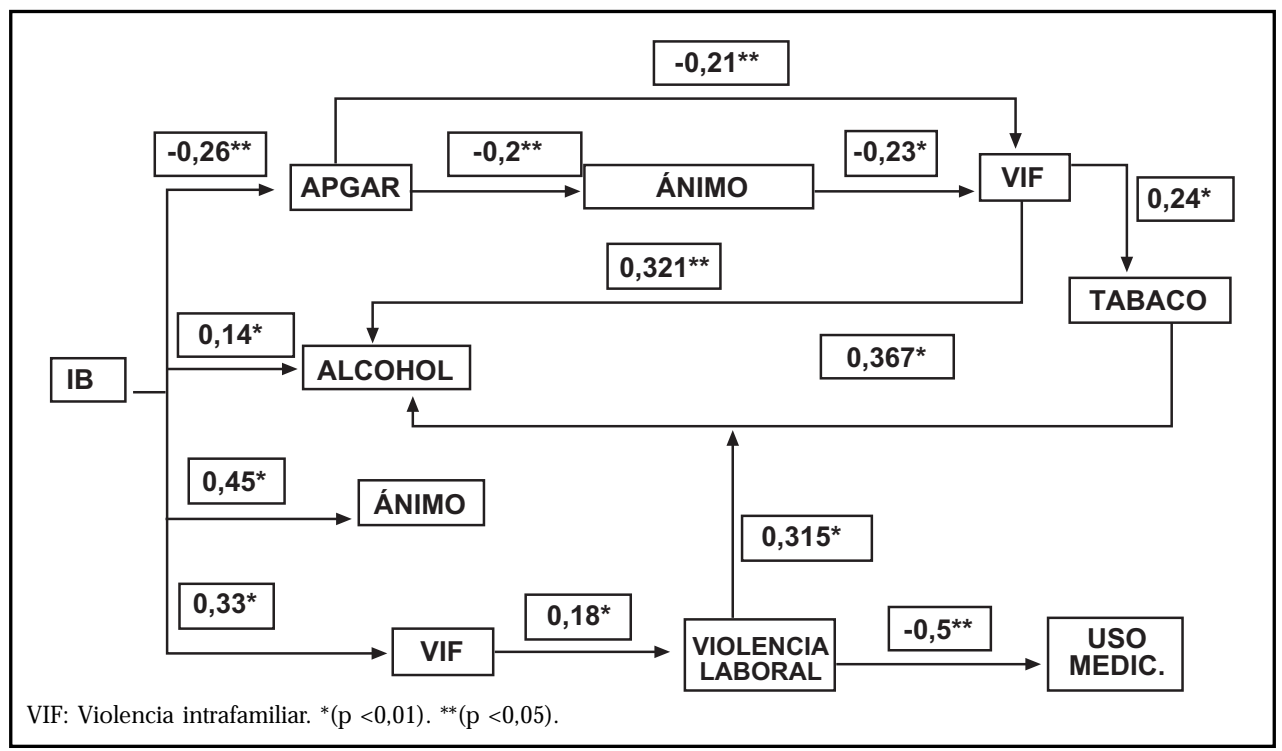

Figura 1. Relación entre IDB y las variables asociadas. 
que trabaje en la atención primaria lo utilice durante la consulta con pacientes hipertensos, derivando a control médico para precisar el diagnóstico sólo en aquellos casos en que se sospeche efectivamente la presencia de un cuadro depresivo.

En el presente estudio, esta escala mostró sólo $9,5 \%$ de falsos positivos. Del total de usuarios que tuvieron puntajes sugerentes de depresión en el Inventario de Depresión de Beck, 100\% obtuvo más de 1 punto en la Escala de Factores de Riesgo del MINSAL, lo que indica que este instrumento, de fácil aplicación, es altamente sensible para detectar a las personas que requieren de una entrevista de diagnóstico y el inicio del plan terapéutico.

Por otro lado, uno de los hallazgos de este estudio fue la alta correlación entre los puntajes del APGAR familiar y los puntajes del IDB. Este es un instrumento de aplicación rápida y que no requiere de personal entrenado, por lo que se sugiere implementar su uso en pacientes en que se presente la condición de comorbilidad entre hipertensión arterial y depresión mayor para así poder instalar un plan de abordaje familiar integral que contribuya a moderar el efecto de esta variable sobre el curso del cuadro clínico.

Por último, los resultados son concordantes con los hallazgos de otros investigadores ${ }^{21,22}$ al revelar una alta correlación entre el consumo de alcohol y tabaco y los síntomas depresivos, relación que interfiere con la instalación de medidas no farmacológicas en el tratamiento de la hipertensión arterial.

En síntesis, la alta prevalencia de depresión mayor en población hipertensa obliga a los profesionales de la salud a estar atentos a las

\section{REFERENCIAS}

1. PUC-MINSAL (2004). Encuesta Nacional de Salud 2003. Disponible en de http://epi.minsal.cl/epi/ $\mathrm{html} /$ invest/ENS/ENS.htm [consultado 18 de agosto de 2008].

2. Ministerio de SAlud. Guía Clínica de HTA primaria o esencial en personas de 15 años o más. Santiago, Chile: MINSAL; 2005.

3. ROJAS P, MONTERO J, MOORE P. Hipertensión Arterial. Temas de Medicina Ambulatoria. Disponible en: http:// manifestaciones clínicas que sugieran la presencia de depresión mayor en este grupo de usuarios. El uso de la Escala de Factores de Riesgo se constituye como un instrumento de pesquisa de fácil aplicación y que requiere muy poca inversión tanto en tiempo como en capacitación del personal, por lo que puede ser incluida dentro de los elementos considerados en el control habitual de estos usuarios. El diagnóstico precoz y la instauración de un plan terapéutico de abordaje eficiente, adecuado y a tiempo repercutirá directamente en la calidad de vida de los mismos, disminuyendo el riesgo de complicaciones cardiovasculares y finalmente influyendo directamente en la disminución de los costos en salud de la atención primaria. Las Garantías Explicitas en Salud consideran intervenciones psicosociales individuales y grupales, además del tratamiento farmacológico, para ambos cuadros clínicos ${ }^{2,4}$, condición que permite diseñar estrategias de intervención conjunta de distintas duplas profesionales para intervenir precozmente y sobre los factores de riesgo modificables en cuadros de baja complejidad y derivar de manera oportuna al especialista en salud mental en caso necesario.

De esta manera se puede contribuir de forma eficiente a equilibrar las demandas de salud de la población beneficiaria lo que repercutirá en aumento de la satisfacción de los usuarios y una mejoría en la capacidad resolutiva de los centros de salud, lo que a su vez permite desarrollar un mayor número de acciones de promoción y prevención, donde el usuario y la comunidad asuman un rol activo como agentes de cambio de su propia situación de salud, sin que se produzca deterioro en la solución oportuna y eficiente de la demanda por las enfermedades.

escuela.med.puc.cl/publ/medamb/hipertensionarterial.html [consultado el 2 de agosto de 2008].

4. Ministerio de SALUd. Guía Clínica para la Atención Primaria: La Depresión, Santiago, Chile: MINSAL; 2001.

5. Vicente B, Kohn R, Saldivia S, Rioseco P. Carga del enfermar psíquico, barreras y brechas en la atención de Salud Mental en Chile. Rev Méd Chile 2007; 135: 1591-9.

6. Minoletti A, López C. Plan nacional de salud mental y psiquiatría. Santiago, Chile: Unidad de salud mental, MINSAL; 2000.

ARtíCULO DE

INVESTIGACIÓN 
7. Grewen M. Depressive symptoms are related to higher ambulatory blood pressure in people with a family history of hypertension. Psychosom Med 2004; 66: 9-16. Disponible en: www.psychosomaticmedicine.org/cgi/reprint/66/1/9 [consultado el $10 \mathrm{de}$ marzo de 2007].

8. Araya M, Madariaga C, Ureta C, Tomé M, Bustos C. La depresión mayor como un nuevo factor de riesgo en la cardiopatía coronaria en Chile. Rev Méd Chile 2002; 130: 1249-56.

9. Broadiey AJ, Frenneaux MP, Moskvina V, Jones CJ, Korszun A. Baroreflex sensitivity is reduced in depression. Psychosom Med 2005; 67: 648-51. Disponible en: http://www.ncbi.nlm.nih.gov.ezproxy.puc.cl/entrez/ query.fcgi:db=PubMed\&tem="Psychosom+Med"[Joumal] [consultado el 12 de marzo de 2007].

10. Sayers S. Depression and Heart Disease. Psychiat Ann 2004; 34: 282-9.

11. Bogner HR, De VRies HF. Integration of depression and hypertension treatment: a pilot randomized controlled trial. Ann Fam Med 2008; 6: 295-301. Disponible en: http://www.annfammed.org/cgi/reprint/6/4/295 [consultado el 31 de octubre de 2008].

12. Guck T, Kavan M, Elsasser G, Barone J. Assessment and treatment of depression following myocardial infarction. Am Fam Physician 2001; 64: 641-9. Disponible en: www.aafp.org/afp/20010815/641.pdf [consultado el 5 de diciembre de 2007].

13. REMCK R. Diagnosis and management of depression in primary care: A clinical update and review. CMAJ 2002; 167: 1253-57. Disponible en: www.cmaj.ca/cgi/reprint/ 167/11/1253 [consultado el 7 de diciembre de 2007].

14. SANZ J, VÁsQuez C. Fiabilidad, validez y datos normativos del Inventario de Depresión de Beck. Psicothema, 1998; 10: 303-18. Disponible en: www.ucm.es/ info/psisalud/carmelo/PUBLICACIONES_pdf/1998BDI\%20en\%investigacion\%20psicopatologica.pdf [consultado el 17 de diciembre de 2006].
15. Aliaga J, Rodríguez de los Ríos L, Ponce C, Frisancho A, EnRíquez J. Escala de desesperanza de Beck (BHS): adaptación y características psicométricas. Rev Investig Psicol 2006; 9: 69-79. Disponible en: http:// sisbib.unmsm.edu.pe/Bvrevistas/Investigación_Psicologia/v09_n1/pdf/a05v9n1.pdf [consultado el 29 de mayo de 2008].

16. Romero C. Depresión y enfermedad cardiovascular. Rev Urug Cardiol 2007; 22: 92-109. Disponible en: www.scielo.edu.uy/scielo.php?script=sci arttext\&pid=S0797-00482007000200004\&Ing=es\&nrm=iso [citado 29 mayo 2008].

17. Lasa L, Ayuso-Mateos J, Vázquez-Barquero J, DiezManRique F, Dowrick C. The use of the Beck Depression Inventory to screen for depression in the general population: a preliminary analysis. J Affect Disord 2000; 57: 261-5.

18. Purebl G, Birkás E, Csoboth C, Szumska I, Kopp M. The Relationship of Biological and Psychological Risk Factors of Cardiovascular Diseases. Behav Med 2006; 31: 133-9.

19. Appeis A, Kop WJ, Schouten E. The nature of the depressive symptomalogy preceding myocardial infarction. Behav Med 2000; 26: 86-9. Disponible en: www.ncbi.nlm.nih.gov/pubmed/11147294 [consultada el 11 de agosto de 2006].

20. Nuyen J, Volkers AC, VerhaAk PF, Schellevis FG, Groenewegen PP, Van Den Bos GA. Accuracy of diagnosing depression in primary care: the impact of chronic somatic and psychiatric co-morbidity. Psychol Med 2005; 35: 1185-95.

21. PiASECKI M. Smoking, nicotine, and mood. En: Piasecki M, Newhouse PA editors. Nicotine in psychiatry. Washington, DC: American Psychyiatric Press; 2000; 131-47.

22. Becoña E, Miguez MC. Consumo de tabaco y psicopatología asociada. Psicooncología 2004; 1: 99-112. Disponible en: http://www.ucm.es/BUCM/revistas/ psi/16967240/articulos/PSIC0404110099A.PDF [consultado el 26 de mayo de 2008]. 